(see R. Aebersold and M. Mann Nature 422, 198-207;2003). "If you want to just do protein identification in fairly normal organisms, such as yeast, humans and mice, where the genomes are known, then that's pretty straightforward," says Ron Bonner, director of applied research at mass spectrometer developers MDS Sciex in Concord, Canada.

\section{Caught in a trap}

Mass spectrometers consist of an ion source, a mass analyser and a detector. Of the four main types of mass analyser currently in use (ion trap, time-of-flight, quadrupole, and Fourier transform ion cyclotron), the workhorse has been the standard three-dimensional (3D) ion trap. "They're relatively cheap, they're extremely robust and they have good performance, but the mass accuracy and mass resolution is really not all that great," says Ruedi Aebersold of the Institute for Systems Biology in Seattle, Washington. "Sensitivity-wise, they're actually very good."

The more recent linear, or two-dimensional, ion traps (see'Mass spectrometry goes mainstream', page 581) have better trapping efficiency and increased ion capacity, which gives them greater resolution, sensitivity and mass accuracy. Electrospray ionization, which ionizes the analytes out of a solution and so can be coupled to liquid-based separation systems, is used in combination with a variety of mass analysers, particularly ion traps. Time-of-flight (TOF) analysers measure the mass of intact peptides with high accuracy and resolution and are often used for high-throughput protein identification

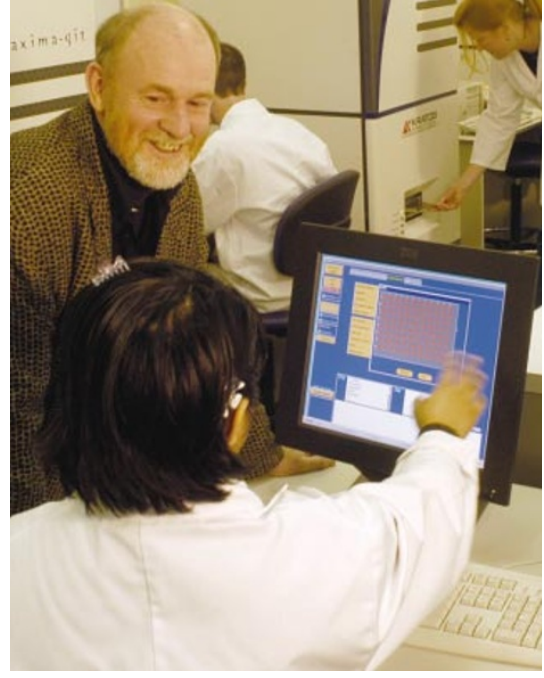

Keith Williams says that providing start-to-finish solutions is key.

by peptide mapping (peptide mass fingerprinting). In these machines, the ionization method is typically matrix-assisted laser desorption/ionization (MALDI).

The novel Sensitizer peptide-labelling reagent developed by Proteome Sciences of Cobham, UK, is aimed at improving the sensitivity of MALDI-TOF and SELDI (surfaceenhanced laser desorption/ionization)-TOF MS systems, in particular, without requiring modifications to instruments or data-analysis software. Increasing the number of proteins identified by peptide mass fingerprinting should mean that fewer samples require full de novo sequencing using the more time-consuming tandem MS (MS/MS).

The buzz this year is over Fourier transform MS (FTMS), with two hybrid machines specifically targeted at proteomics coming onto the market (see 'Mass spectrometry goes mainstream', page 581). FTMS was used to identify more than $60 \%$ of the predicted proteome of the bacterium Deinococcus radiodurans, the most complete coverage of any proteome yet. And although the analysis of post-translational modifications such as glycosylation and phosphorylation using MS is still something of a challenge, FTMS was able to identify almost every post-translational modification of a $29 \mathrm{kDa}$ protein.

The well-established technique of twodimensional gel electrophoresis (2DE) has been used most commonly for separating protein mixtures in preparation for MS. And this is likely to continue in the near future. To some extent, "it's because old habits die hard", says Aebersold. After staining and image analysis, selected spots from the gel are excised, digested and the peptides analysed by MS. Despite attempts at automation (see 'Look, no hands', below), 2DE is likely to remain fairly low-throughput. It also requires relatively large amounts of sample (an issue with clinical material) and in the past has failed to detect low-abundance proteins reliably. Pre-fractionation of the sample, or the use of more sensitive staining methods and larger-format gels, can enhance detection of low-abundance proteins.

The shortcomings of 2DE-MS have fuelled interest in eliminating the need for gels. Liquid

\title{
LOOK, NO HANDS
}

A recurring theme in proteomics is the demand for greater automation to increase efficiency and reproducibility. But to improve somebody's process, "you first have to convince them they've got one", says John Hobbs, strategic market manager for Beckman Coulter in Fullerton, California. Beckman Coulter has modified and repositioned some of its products under the name ProteomeLab. The products are very "solutions-orientated", says Hobbs. With the PF 2D automated, two-dimensional protein-fractionation system, for example, the company supplies the chemistry, the hardware, the software and the methods, and "we basically tell the customer don't mess with it", he says. The PF 2D uses chromatofocusing followed by non-porous reverse-phase chromatography for high-resolution separation of proteins from complex mixtures. Fraction collection from the first dimension is controlled by an in-line $\mathrm{pH}$ monitor, with automatic injection of the fractions into the second dimension. Liquid fractions can be stored or the eluent connected directly to electrospray ionization-mass spectrometry. Beckman Coulter is working with mass spectrometer vendors to ensure compatibility with MALDI (matrix-assisted laser desorption/ionization) plate spotters.

Although non-gel-based methods coupled with mass spectrometry (MS) are gaining acceptance, the most commonly used approach is still a combination of two-dimensional gel electrophoresis (2DE) and MS. Twodimensional gel electrophoresis is difficult to automate, but NextGen Sciences of Huntingdon, UK, will soon be shipping a fully automated 2DE platform - a2DE. Immobilized pH gradient strips (up to $30 \mathrm{~cm}$ ) are run in parallel in the first dimension and three large-format SDS-PAGE gels in the second, and all steps between sample injection and SDS-PAGE are automated. Future versions will allow the same degree of optimization in the first dimension as currently offered in the second dimension, says chief executive Kevin Auton. Then "you can really start to zoom in on certain isoelectric points, as well as molecular-weight ranges", he says.

For about US\$2 million, Proteome Systems in North Ryde, Australia, sells a start-to-finish automated proteomics solution called ProteomIQ, launched last year - or you can buy the components separately. It comprises kits and instruments for sample preparation and protein separation, ShimadzuBiotech's Axima CFR Plus MALDI-TOF (time-of-flight) mass spectrometer and bioinformatics capabilities. The Xcise gel-processing robot, developed jointly with Shimadzu-Biotech of Kyoto, Japan, bundles the functions of gel imaging, precision spot cutting, protein digestion and peptide purification, and deposition onto a target plate into one instrument. Also developed with Shimadzu-Biotech, the novel Chemical Inkjet Printer (ChIP) uses piezoelectric printing technology to dispense minute amounts of reagents onto selected protein spots electroblotted from 2D gels onto membranes. After processing, the membrane can be placed directly into the mass spectrometer for peptide analysis. ChIP not only cuts down on liquid-handling steps but also offers the option of archiving samples for future analysis. 